\title{
Molecular Imaging in Pancreatic Cancer - A Roadmap for Therapeutic Decisions
}

\author{
Gabriela Kramer-Marek ${ }^{\dagger}$, Jesse Gore, and Murray Korc ${ }^{\dagger}$ \\ Indiana University School of Medicine, Indianapolis, IN
}

\begin{abstract}
Pancreatic ductal adenocarcinoma (PDAC) is a deadly cancer characterized by multiple molecular alterations, the presence of an intense stroma, poor perfusion, and resistance to therapy. In addition to standard imaging techniques, experimental imaging strategies, such as those utilizing molecular probes, nanoparticle-based agents, and tagged antibodies are actively being explored experimentally. It is hoped that advances in these technologies will allow for detecting PDAC at an early stage, and could serve to validate experimental therapies, rapidly identify non-responders, and assist in the design of novel therapeutic strategies tailored to the patient's molecular profile.
\end{abstract}

\section{Keywords}

PDAC; molecular imaging; ultrasonography

\section{PDAC: an overview}

Pancreatic ductal adenocarcinoma (PDAC) is the fourth leading cause of cancer-related death in United States with an overall 5-year survival of 5\% [1]. Despite remarkable advances in the past two decades that have greatly improved our understanding of the pathobiology of PDAC, overall median survival in PDAC is a dismal 8 to 12 months in patients with localized disease, and 4 to 6 months in patients with metastatic disease [2]. These alarming statistics are due to multiple factors, including the paucity of specific symptoms even when PDAC is already well-established, the absence of specific and sensitive markers that would allow for early stage diagnosis, and the advanced stage at presentation which precludes resection. Moreover, there is a high recurrence rate following resection, and most therapeutic approaches are of limited effectiveness due to intrinsic chemoresistance and radioresistance of the cancer cells, and the presence of a dense stroma rich in collagens and mucins that combine to prevent therapeutic agents from penetrating the tumor mass. Together, these alterations exacerbate response to therapy [3, 4], and even new combinatorial treatment regimens only prolong survival for a few months [5].

(C) 2013 Elsevier Ireland Ltd. All rights reserved.

"Corresponding author: Murray Korc, MD, School of Medicine, Indiana University, Walther Hall R3 C528, 980 West Walnut Street, Indianapolis, IN 46202, USA, mkorc@iu.edu.

$\dagger$ Current address: The Institute of Cancer Research, Division of Radiotherapy and Imaging, 15 Cotswold Road, Sutton, Surrey, SM2 $5 \mathrm{NG}$, United Kingdom

Conflicts of interest

None declared

Publisher's Disclaimer: This is a PDF file of an unedited manuscript that has been accepted for publication. As a service to our customers we are providing this early version of the manuscript. The manuscript will undergo copyediting, typesetting, and review of the resulting proof before it is published in its final citable form. Please note that during the production process errors may be discovered which could affect the content, and all legal disclaimers that apply to the journal pertain. 
This review will focus on the potential role of imaging modalities such as computed tomography (CT), magnetic resonance imaging (MRI), ultrasonography (US), positron emission tomography (PET), and optical imaging that could be combined with molecular profiling to assist in the identification of novel biomarkers to improve diagnosis, predict therapeutic responses, and identify new molecular targets, thereby potentially pointing to strategies that will improve the management and survival of PDAC patients.

\section{Molecular Pathogenesis of PDAC}

PDAC is believed to arise from precursor lesions that develop into invasive carcinoma through a multistep carcinogenic process. The most common pre-neoplastic lesion that has been found in approximately $80 \%$ of patients with PDAC is pancreatic intraepithelial neoplasia (PanIN) [6]. PanIN lesions are classified as PanIN1A, PanIN1B, PanIN2 and PanIN3, depending on the grade of architectural and nuclear atypia. Thus, PanIN1A lesions consist of columnar cells forming ductal-like structures and exhibit an abundance of mucin which stains intensely with Alcian blue (Fig. 1A). PanIN1A lesions are also seen in patients with chronic pancreatitis and in the elderly, but they are not necessarily pre-malignant [7]. Similarly, PanIN1B lesions are not necessarily pre-malignant and are distinguished from PanIN1A by the presence of papillary structures (Fig. 1B, C). PanIN progression is associated with increasing cellular and nuclear atypia, a cribriform appearance, varying degrees of loss of polarity, and luminal budding. PanIN3 lesions represent a carcinoma in situ, and may harbor mitotic figures and exhibit local invasion.

PanIN may arise in regions of acinar-to-ductal metaplasia (ADM), and it has been suggested that progenitor cells giving rise to the acinar cell lineage give rise to ADM and PanIN lesions, and ultimately PDAC [8, 9]. Other precursor lesions include intraductal papillary mucinous neoplasms (IPMN) and mucinous cystic neoplasms (MCN), which harbor specific molecular alterations [10].

PDAC is characterized by a high frequency of major driver mutations, including KRAS (95\%), CDKN2A (90\% mutated, 10\% epigenetically silenced), TP53 (70\%), and SMAD4 $(55 \%)$, as well as by low-frequency driver mutations that yield a unique tapestry of gene alterations in any individual with PDAC [11, 12].

PDAC is also characterized by constitutive activation of pro-survival pathways including STAT3, NFkB, and AKT, which serve to enhance apoptosis resistance. In addition, there is excessive production of receptor tyrosine kinases (RTKs) and ligands. For example, the EGF receptor (EGFR) is a pivotal RTK that is overexpressed at both the mRNA and protein level in 50-60\% of resectable PDACs [13], whereas the human EGF receptor 2 (HER2) is overexpressed in $45-70 \%$ of PDAC cases [14], and this overexpression may be associated with more aggressive disease and poor clinical outcome [15]. Furthermore, HER3, which has the capacity to excessively activate PI3K/AKT due to phosphorylation of its 7 tyrosine residues upon receptor heterodimerization, is overexpressed in $60 \%$ of resectable PDAC, and this overexpression correlates with decreased survival irrespective of ligand levels [16].

Recent studies indicate that pancreatic-cancer cells carry an average of 63 genetic alterations per cancer, which can be grouped to 12 core signaling pathways [12]. In addition, PDAC is associated with increased cyclin D1 expression, aberrant activation of transforming growth factor-beta (TGF- ) pathways in conjunction with increased expression of TGF- isoforms, and a hypoxic microenvironment rich in inflammatory cells and cytokines that promote cancer growth [7]. In addition, there is inappropriate reactivation of developmental pathways, such as Hedgehog (Hh), notch and wnt/ -catenin whose roles have been comprehensively summarized $[6,17,18]$. Taken together, these observations underscore the 
complexity of the genetic and molecular mechanisms that drive PDAC aggressiveness and the need for a personalized approach in PDAC treatment. Moreover, they point to novel candidates for drug and imaging agent targeting. Such an approach has been shown to be successful using trastuzumab and affibody molecules in HER2-overexpressing breast cancer cells [19-21].

\section{PDAC Microenvironment}

PDAC is a highly desmoplastic cancer. The prominent stroma is a complex structure that consists of proliferating pancreatic stellate cells (PSC), cancer-associated fibroblasts, degenerating acinar cells, foci of aberrant micro-angiogenesis, and varying types of inflammatory cells [22]. The stromal compartment plays an active role in promoting invasion and growth of PDAC cells, and at the same time, is a physical barrier for drug delivery [23]. PSCs have a strategic role in stroma formation, and are involved in tumor growth, invasion and metastasis [24, 25]. Upon activation, they synthesize and release growth factors and produce large amounts of extracellular matrix (ECM) proteins leading to ECM remodeling, and finally, to a hypovascular and hypoxic stroma [10].

The role of angiogenesis in PDAC remains controversial. Early data correlating microvessel hot spots in PDAC with known modulators of angiogenesis, as well as analysis of angiogenesis in orthotopic mouse models of PDAC, have suggested that PDAC is angiogenesis-dependent, but angiogenesis inhibitors have failed in clinical trials [26-28]. With the establishment of genetically engineered mouse models (GEMs) of PDAC, it became possible to gain a better understanding of the role of key genes and pathways involved in PDAC initiation and progression, identify new predictive biomarkers, validate novel therapeutic approaches, and evaluate the mechanisms responsible for chemoresistance. A number of murine PDAC (mPDAC) models have been generated by targeting a conditionally mutated Kras allele ( $\left.L S L-K_{r a s} G 12 D\right)$ to pancreatic progenitors, using Cre recombinase driven by either Pdxl or Ptfla promoters [42]. This so-called KC (Kras G12D allele and Cre-driven recombinase) GEM is characterized by slow PanIN progression to invasive mPDAC and a low incidence of metastasis (average latency 1 yr) [29, 30]. By contrast, combining oncogenic Kras with inactivation of tumor suppressor genes, such as Ink4a, p53 and SMAD4 markedly accelerated PanIN progression to mPDAC [30-32]. Moreover, the dense stroma that occurs in these GEMs exhibits features that resemble human disease. For example, mPDAC arising in Pdx1-Cre/Kras G12D/p53 ${ }^{\mathrm{R} 172 \mathrm{H}}$ mice, which harbor heterozygous conditional mutant alleles of KRAS and $p 53$, exhibit marked hypovascularity and aberrant vascular architecture [33]. The dense stroma and the hypovascularity are important contributors to chemoresistance, and targeting the stromal hedgehog pathway attenuates desmoplasia and improves tumor vascularization leading to increased drug delivery [23]. However, in an oncogenic Kras-driven GEM that lacks p16 ${ }^{\text {Ink4a }}$ and glypican-1 (GPC1), mPDACs display attenuated angiogenesis and growth [34]. This is due to loss of the pro-angiogenic actions of heparin-binding growth factors that depend on the presence of GPC1 [34]. Thus, in spite of the paucity of blood vessels in this mPDAC model and its marked desmoplasia, tumor angiogenesis can nonetheless contribute to mPDAC biological aggressiveness.

\section{The Need for Imaging Advances in PDAC}

Molecular imaging plays an important role in cancer management and has been successfully employed for tumor detection and characterization, staging, and response to therapeutic intervention. The primary advantage of in vivo imaging is the ability to characterize malignancies in a non-invasive way, and at the same time, provide quantitative data. 
The use of imaging in clinical practice in PDAC has proven challenging due to the retroperitoneal location of the pancreas. Currently, the most common modalities that have been used to image the pancreas include CT, MRI and endoscopic ultrasonography (EUS), but these methods are limited to diagnosis and staging. Recently, however, radionuclide imaging using PET and ${ }^{18}$ F-FDG has shown promise. Moreover, optical technologies such as near infrared imaging and multispectral optoacoustic imaging that are mainly used experimentally, may be translated into the clinic in the near future.

Each of these imaging modalities have variable sensitivities, and enable visualization of malignant tissues from different perspectives, such as tissue density, metabolism, vascularity and/or water content (Table 1). While conventional radiological techniques improve the specificity of PDAC diagnosis, they are limited by their ability to detect PDAC at a resectable stage that precedes perineural invasion, vascular involvement and the formation of distant metastases. Therefore, there is a dire need to improve their sensitivity, specificity and accuracy to improve early stage diagnostics and identify specific biomarkers and molecular probes.

The development of preclinical models that recapitulate the human disease in vivo has aided in the identification of novel biomarkers, and the development of screening strategies to improve clinical outcome. Among the various biomarker candidates and diagnostic tools identified and studied thus far standouts include integrin ligands, plectin-1, cathepsin activity, nanoparticle-based agents, antibodies and contrast agents, all of which have shown promising results in experimental settings. These probes used in conjunction with molecular imaging techniques could serve as the guides for i) detection of early cancer, ii) validation of putative drugs and different treatment regimens, and iii) early identification of nonresponders. Such approaches would lead to patient stratification through a precise personalized approach for targeting patient-specific pathway alterations in the tumor. Next, we highlight new advances in PDAC imaging, the advantages and disadvantages of different modalities used to detect primary and metastatic lesions, and describe their potential usefulness as predictive tools for targeted therapy.

\section{Imaging modalities}

Endoscopic ultrasound (EUS) involves the use of a high-frequency ultrasound (US) transducer that was developed in the early 1980s specifically to better visualize the pancreas [35]. US relies on the reception, analysis and display of acoustic signals produced by reflection or backscatter of sound above the audible frequency range for humans $(>20 \mathrm{kHz})$. In contrast to other imaging modalities, it offers significant advantages such as real-time imaging acquisition, wide availability, safety and low cost. This technique, however, is limited by operator dependence, resulting in variable accuracy ranging from 57\% to $81 \%$ [36]. Introduction of Doppler-based methods for assessing the direction and velocity of blood flow have expanded the diagnostic capabilities of US, but Doppler cannot be used to assess microvascular blood flow of tissues.

EUS can provide reliable information about the size and local extent of the primary tumor. By guiding the probe in close proximity to the pancreas, clinicians can image the whole organ, and biopsy suspicious masses under direct visualization thereby obtaining detailed, high resolution images and tissue samples for pathological evaluation. The development of electronic radial and linear transducers as well as color Doppler capabilities has also improved the accuracy of diagnosis and staging, establishing EUS as the procedure of choice for the evaluation of patients with pancreatic cancer [37]. Currently, the sensitivity of EUS for detecting pancreatic lesions ranges from $85 \%$ to $99 \%$, which is higher than the sensitivity of CT in detecting small pancreatic nodules [38]. Furthermore, the method is able 
to detect focal lesions as small as $2-3 \mathrm{~mm}$ and can be as accurate as CT in detecting liver metastases [39]. The bulk of the literature supports its superiority over CT, MRI and PET for pancreatic tumor detection and local staging [40, 41]. However, in order to predict tumor resectability, the combination of CT and EUS proved to be the most accurate method compared to either modality alone [42]. Moreover, EUS-guided Fine Needle Aspiration (EUS-FNA) allows for tissue sampling and cytological evaluation of the primary tumor and adjacent suspicious lymph nodes that are difficult to detect by CT or MRI. This procedure has had a major impact on therapeutic management of the patients by providing a definite tissue diagnosis, with an overall $85 \%-90 \%$ sensitivity and almost $100 \%$ specificity $[43,44]$.

The advent of contrast medium agents has enhanced the ability to obtain essential quantitative information relating to tissue vascularity, perfusion and even endothelial wall function. The most commonly used contrast agents are gas-filled microbubbles. Their purely vascular localization makes them ideal for the development of targeted contrast agents, especially in relation to receptors expressed on the endothelial lining of vessels. Currently, contrast-enhanced US imaging is only used in pre-clinical studies. For example, microbubble-enhanced US was recently used as a method to noninvasively monitor the functional effects of an anti-angiogenic agent in GEMs of PDAC [45]. However, ongoing research indicates it might be soon translated into clinical trials.

$C T$ imaging is based on the measurements of the attenuation of x-rays passing through an object and provides excellent anatomic context. It also supports separately acquired emission images of the distribution of radiolabeled agents, and increases their accuracy by providing attenuation correction of the emission intensities. Currently, it is widely used for initial diagnosis and staging of pancreatic cancer and liver metastases [46]. Introduction of dual-phased multi-detector CT (MDCT), which uses a wider x-ray beam, narrower detector collimation, and more rapid table translation, resulted in much faster image acquisition during different temporal phases compared with conventional CT [47]. Additionally, it has allowed for imaging of larger volumes of pancreatic tissue while providing useful information on arterial and venous phases, and better delineation of the main pancreatic duct and small intra-parenchymal masses. Moreover, introduction of more sophisticated reconstruction algorithms have resulted in better contrast and spatial resolution images that together have improved detection and staging of PDAC.

To date MDCT has the highest accuracy in assessing the extent of the primary tumor, locoregional extension, vascular invasion, distant metastasis, tumor-node-metastasis (TNM) stage and tumor resectability. MDCT has been used in the preoperative staging of PDAC, with a sensitivity and specificity for predicting resectability of $96 \%$ and $33 \%$, respectively [48]. Despite its sensitivity for detecting potentially respectable tumors, MDCT is rather nonspecific, therefore, detection of cystic lesions or lesions $<2 \mathrm{~cm}$ might be challenging by this technique [49]. Moreover, this technique cannot reliably differentiate between benign and malignant lesions. Thus, other modalities with higher specificity should be considered for PDAC staging. Recently, perfusion CT, which quantifies tissue perfusion following contrast agent administration, has shown promise in detecting pancreatic tumors with higher specificity, and differentiating low-grade from high-grade PDAC [50, 51]. However, the accuracy of this method depends on hypovascular PDACs that exhibit diminished levels of contrast enhancement compared to the surrounding pancreas.

PET imaging is based on the decay of a radioisotope, which emits a positron from the nucleus that subsequently annihilates with an electron to produce two high-energy (511 $\mathrm{keV}$ ) photons that propagate in nearly opposite directions. The information provided by this modality, including tissue function, blood flow, metabolic activity and receptor expression, is useful for diagnosis, staging, treatment and management of cancer. PET is particularly 
helpful in the evaluation of distant metastases, and in instances of an equivocal CT or MRI diagnosis. Moreover, unlike anatomical imaging modalities that measure changes in tumor volume, PET allows treatment effects to be directly monitored at earlier time points before any tumor volumetric changes are visible on standard CT or MR images.

The tracer that has had a major impact on clinical PET imaging, and is considered a gold standard is ${ }^{18} \mathrm{~F}$-fluorodeoxyglucose $\left({ }^{18} \mathrm{~F}\right.$-FDG). It is an analog of glucose taken up by the cancer cells through the glucose transport pathway, but it cannot be metabolized and therefore remains trapped within metabolically active tissues. The normal pancreas has low glucose utilization compared with tumors. Therefore, foci of abnormal ${ }^{18} \mathrm{~F}-\mathrm{FDG}$ uptake can be easily visualized as focal areas of increased activity (Fig. 2). Gambhir et al., in their tabulated review, demonstrated that in 387 PDAC patients, the average sensitivity and specificity of ${ }^{18} \mathrm{~F}-\mathrm{FDG}$ were $94 \%$ and $90 \%$, respectively, compared to $82 \%$ and $75 \%$ for CT [52]. Similar results were obtained by Keogan et al., who reported that this non-tumorspecific molecule has high sensitivity (88-92\%) and specificity (83\%-85\%) for diagnosing primary PDAC [53].

The presence of focal ${ }^{18} \mathrm{~F}-\mathrm{FDG}$ activity also provides a significant advantage for diagnosing small metastatic lesions. However, since glucose metabolism is not specific for malignant processes, physiologic ${ }^{18} \mathrm{~F}-\mathrm{FDG}$ uptake can be found in normal tissues (brain, muscles, salivary gland, myocardium, urinary tract) as well as in inflammatory and benign lesions, which might lead to false positive or false-negative findings. As a result, chronic pancreatitis and PDAC may have a similar appearance [54]. Fendrich et al., showed strong ${ }^{18} \mathrm{~F}-\mathrm{FDG}$ uptake in mPDAC, but a weak signal in the pancreata of mice with PanIN lesions, suggesting that glucose metabolism increases during PDAC progression [55]. These findings undoubtedly support further exploration of PET imaging and support the statement that, in general, it is the combination of PET with CT and/or MRI that improves the sensitivity for diagnosing a malignancy compared with each modality alone.

The role of ${ }^{18} \mathrm{~F}-\mathrm{FDG}$ is staging, however, is questionable. Poor spatial resolution limits the local staging of PDAC, and primary tumors should be evaluated by modalities that image the tumor relative to adjacent organs and vascular structures, such as EUS, CT, MRI. On the other hand, PET is useful for identifying distant metastases [56]. Thus, the sensitivity and specificity of ${ }^{18} \mathrm{~F}$-FGD-PET for detecting hepatic metastases was $95 \%$, only missing liver lesions $<1 \mathrm{~cm}$ [57]. The impact of ${ }^{18} \mathrm{~F}-\mathrm{FDG}$ on patient management is not clearly evident. There are no accepted screening tests that would identify early stage PDAC and there is no justification for using ${ }^{18} \mathrm{~F}-\mathrm{FDG}$ as a population screening tool. Moreover, by the time patients undergo any form of imaging, there is an $80 \%$ chance that the disease is at an advanced stage and has become unresectable. Therefore, there is a dire need to develop imaging platforms with enhanced abilities to visualize specific molecular markers at the initial stages of PDAC development.

MRI techniques involve manipulation of nuclear magnetic dipole moments by means of externally applied magnetic fields, and the subsequent recording and mathematical analysis of radio signals emitted from the nuclei in response to these manipulations. This imaging modality can provide information about tissue structure, perfusion and function (Magnetic Resonance Spectroscopy). One of the key advantages of MRI over CT is superior contrast among soft tissues. Moreover, MRI has multiplanar capabilities that are absent from CT. The sensitivity of MRI is low but can be improved by introducing contrast agents that magnetically modify the environment affecting either $\mathrm{T} 1$ or $\mathrm{T} 2$ relaxation time constants, proton density, or nuclear polarization. By contrast, the spatial resolution of MRI is extremely good $(\mathrm{m})$, and is not limited by excitation wavelength or the diffraction limit of photons. Some consider MRI to be the best first line noninvasive imaging modality in 
suspected PDAC patients, since it can evaluate pancreas (benign vs. malignant), vasculature and pancreaticobiliary ducts in a single examination [37]. Due to its high soft tissue contrast and more extensive types of data that can be acquired, some argue that MRI is even more predictable than CT in evaluation of pancreatic tumors, especially in detecting small noncontour-deforming lesions and subtle liver metastasis [58].

Diffusion-weighed imaging (DWI) is a relatively new MRI technique that reflects changes in water mobility caused by interactions with cell membranes and macromolecules, and alterations in the tissue environment. Therefore, DWI provides a tissue contrast that is different from that of conventional T1- and T2-weighted MRI images. Inasmuch as DWI offers quantitative measurements of the diffusivity of water described by the apparent diffusion coefficient (ADC), it represents microcirculation of blood perfusion and the molecular diffusion of water. Therefore, in pancreatic cancer, ADC values are usually lower than in normal pancreatic tissue. Using DWI, Kamisawa et al., evaluated its clinical utility in patients with cancer and autoimmune pancreatitis (AIP), and assessed whether DWI could help differentiate cancer from AIP [59]. They determined that ADC values were significantly lower in AIP than in PDAC and normal pancreas, underscoring the potential of DWI as a diagnostic test in AIP.

Optical imaging is a very sensitive and versatile modality that is based on detecting the transmission of light through biological tissues. Photons traveling through the tissue simultaneously undergo absorption, attenuation and scattering that are wavelength and penetration-depth dependent. Consequently, it is not possible to conduct rigorous quantitative assessments with this technique. Currently, there are several optical imaging technologies, such as bioluminescence imaging (BLI), fluorescence imaging (FLI), diffuse optical tomography (DOT), and optical projection tomography (OPT). All of these imaging modalities are emerging as powerful tools for measuring dynamic metabolic processes and probing protease, protein and enzymatic activity in vivo.

In DOT, diffuse light penetrates tissues at multiple projections yielding tomographic images. Introduction of this technique was a milestone in optical technology, since DOT provides quantitative information about light absorption, scattering and uptake of fluorescence contrast agents. OPT technology was developed more recently for 3D imaging, and has emerged as a powerful tool for visualization of specimen areas with sizes between 1-10 mm. It is essentially, the optical equivalent of X-ray tomography that is based on the acquisition of a sequence of optical transmission images through the sample, which is rotated at several angles. The application of optical imaging to date has been largely investigated in vivo in murine models providing unique insights into disease pathogenesis, drug development and the effects of therapy. In this way, optical imaging already is making a substantial impact on basic and translational medical research.

\section{Imaging Experimental Models}

BLI holds great potential for experimental research purposes primarily as a high throughput, small animal imaging modality. It is based on the self-emission of light from yellow to green wavelengths owing to catalysis of luciferase enzymes that takes place in the presence of luciferin substrates [60]. The advantage of BLI is the minimal background signal and an excellent signal-to-noise ratio (SNR), which makes this technique highly sensitive (Fig. 3). However, due to the fact that it is a two-dimensional planar modality, BLI does not allow for absolute quantification of target signal. FLI refers to the property of certain molecules to absorb light at a particular wavelength and to emit light of a longer wavelength after a brief interval known as the fluorescence lifetime [61]. Both, BLI and FLI modalities using specific promoters to drive reporter gene expression provides the ability to localize and 
measure gene and protein expression, and monitor cell trafficking, tumor growth and responsiveness to new therapies.

In orthotopic pancreatic cancer models, Bouvet et al., used BxPC-3 and MiaPaCa-2 pancreatic cancer cell lines that stably and robustly express GFP to demonstrate external, real-time, whole body and intravital fluorescence imaging of tumor growth and metastasis progression [62]. Also, the near-infrared fluorescently-labeled recombinant protein, PTDODD-HaloTag (POH), allowed for the successful monitoring of HIF-active regions within small localized and metastatic foci in an orthotopic pancreatic mouse model, in which HIF positive cancer cells play a central role during local invasion and metastasis [63]. Fluorescent images combined with BLI identified HIF(+) cancer cells at 9-24 h after intraperitoneal injection of labeled agent [63]. Moreover, in a PDAC GEM, it was recently demonstrated that a cathepsin-activated NIRF probe can be used with the vascular contrast agent fluorescein to differentiate between low-grade and high-grade murine PanINs and early-stage mPDAC in vivo, combining the advantages of IHC and real-time confocal fluorescence laser microscopy in a Kras ${ }^{G 12 D}$-driven mouse model [64].

\section{Conclusions}

Current demands and trends call for new strategies to focus on the discovery of novel biomarkers specific for PDAC that distinguish between different stages and grades of PDAC and perhaps allow for earlier disease detection. Such biomarkers could also serve as prognostic tools or as potential therapeutic drug targets. Improved imaging and screening protocols could allow for the screening of high risk populations, patient-specific treatment selection, and imaging-based therapy monitoring. Taken together, these strategies could ultimately improve therapeutic options in this deadly cancer.

\section{Acknowledgments}

The authors acknowledge the assistance of the Indiana University Simon Cancer Center In Vivo Imaging Core Facility in obtaining BLI images of mice bearing orthotopically implanted human pancreatic tumors. The authors also thank Dr. Gregory Ravizzini in the Department of Nuclear Medicine at the University of Texas MD Anderson Cancer Center for providing the fused PET/CT image. Research reported in this publication was supported by the National Cancer Institute of the National Institutes of Health under award number R37-CA-075059 to M. Korc.

\section{References}

[1]. Raimondi S, Maisonneuve P, Lowenfels AB. Epidemiology of pancreatic cancer: an overview. Nat Rev Gastroenterol Hepatol. 2009; 6:699-708. [PubMed: 19806144]

[2]. Nieto J, Grossbard ML, Kozuch P. Metastatic pancreatic cancer 2008: Is the glass less empty? Oncologist. 2008; 13:562-576. [PubMed: 18515741]

[3]. Erkan M, Reiser-Erkan C, Michalski CW, Kleeff J. Tumor microenvironment and progression of pancreatic cancer. Exp Oncol. 2010; 32:128-131. [PubMed: 21403605]

[4]. Wang Z, Li Y, Ahmad A, Banerjee S, Azmi AS, Kong D, Sarkar FH. Pancreatic cancer: understanding and overcoming chemoresistance. Nat Rev Gastroenterol Hepatol. 2011; 8:27-33. [PubMed: 21102532]

[5]. Preis M, Korc M. Signaling pathways in pancreatic cancer. Crit Rev Eukaryot Gene Expr. 2011; 21:115-129. [PubMed: 22077151]

[6]. Ansari D, Chen BC, Dong L, Zhou MT, Andersson R. Pancreatic cancer: Translational research aspects and clinical implications. World J Gastroenterol. 2012; 18:1417-1424. [PubMed: 22509073]

[7]. Baumgart M, Werther M, Bockholt A, Scheurer M, Ruschoff J, Dietmaier W, et al. Genomic instability at both the base pair level and the chromosomal level is detectable in earliest PanIN lesions in tissues of chronic pancreatitis. Pancreas. 2010; 39:1093-1103. [PubMed: 20531246] 
[8]. Carriere C, Young AL, Gunn JR, Longnecker DS, Korc M. Acute pancreatitis accelerates initiation and progression to pancreatic cancer in mice expressing oncogenic Kras in the nestin cell lineage. PLoS One. 2011; 6:e27725. [PubMed: 22140463]

[9]. Shi G, Direnzo D, Qu C, Barney D, Miley D, Konieczny SF. Maintenance of acinar cell organization is critical to preventing Kras-induced acinar-ductal metaplasia. Oncogene. 2012

[10]. Krautz C, Ruckert F, Saeger HD, Pilarsky C, Grutzmann R. An update on molecular research of pancreatic adenocarcinoma. Anticancer Agents Med Chem. 2011; 11:411-417. [PubMed: 21492076]

[11]. Biankin AV, Waddell N, Kassahn KS, Gingras MC, Muthuswamy LB, Johns AL, et al. Pancreatic cancer genomes reveal aberrations in axon guidance pathway genes. Nature. 2012; 491:399-405. [PubMed: 23103869]

[12]. Jones S, Zhang X, Parsons DW, Lin JC, Leary RJ, Angenendt P, et al. Core signaling pathways in human pancreatic cancers revealed by global genomic analyses. Science. 2008; 321:18011806. [PubMed: 18772397]

[13]. Preis M, Korc M. Kinase signaling pathways as targets for intervention in pancreatic cancer. Cancer Biol Ther. 2010; 9:754-763. [PubMed: 20234186]

[14]. Yamanaka Y, Friess H, Kobrin MS, Buchler M, Kunz J, Beger HG, Korc M. Overexpression of HER2/neu oncogene in human pancreatic carcinoma. Hum Pathol. 1993; 24:1127-1134. [PubMed: 8104858]

[15]. Baselga J, Swain SM. Novel anticancer targets: revisiting ERBB2 and discovering ERBB3. Nat Rev Cancer. 2009; 9:463-475. [PubMed: 19536107]

[16]. Friess H, Yamanaka Y, Kobrin MS, Do DA, Buchler MW, Korc M. Enhanced erbB-3 expression in human pancreatic cancer correlates with tumor progression. Clin Cancer Res. 1995; 1:14131420. [PubMed: 9815939]

[17]. Wong HH, Lemoine NR. Pancreatic cancer: molecular pathogenesis and new therapeutic targets. Nat Rev Gastroenterol Hepatol. 2009; 6:412-422. [PubMed: 19506583]

[18]. Morris JP, Wang SC, Hebrok M. KRAS, Hedgehog, Wnt and the twisted developmental biology of pancreatic ductal adenocarcinoma, Nature reviews. Cancer. 2010; 10:683-695. [PubMed: 20814421]

[19]. Kramer-Marek G, Capala J. Can PET Imaging Facilitate Optimization of Cancer Therapies? Curr Pharm Des. 2012

[20]. Dijkers EC, Oude Munnink TH, Kosterink JG, Brouwers AH, Jager PL, de Jong JR, et al. Biodistribution of 89Zr-trastuzumab and PET imaging of HER2-positive lesions in patients with metastatic breast cancer. Clin Pharmacol Ther. 2010; 87:586-592. [PubMed: 20357763]

[21]. Valabrega G, Montemurro F, Aglietta M. Trastuzumab: mechanism of action, resistance and future perspectives in HER2-overexpressing breast cancer. Ann Oncol. 2007; 18:977-984. [PubMed: 17229773]

[22]. Erkan M, Hausmann S, Michalski CW, Fingerle AA, Dobritz M, Kleeff J, Friess H. The role of stroma in pancreatic cancer: diagnostic and therapeutic implications. Nat Rev Gastroenterol Hepatol. 2012; 9:454-467. [PubMed: 22710569]

[23]. Olive KP, Jacobetz MA, Davidson CJ, Gopinathan A, McIntyre D, Honess D, et al. Inhibition of Hedgehog signaling enhances delivery of chemotherapy in a mouse model of pancreatic cancer. Science. 2009; 324:1457-1461. [PubMed: 19460966]

[24]. Duner S, Lopatko Lindman J, Ansari D, Gundewar C, Andersson R. Pancreatic cancer: the role of pancreatic stellate cells in tumor progression. Pancreatology. 2010; 10:673-681. [PubMed: 21242706]

[25]. Xu Z, Vonlaufen A, Phillips PA, Fiala-Beer E, Zhang X, Yang L, et al. Role of pancreatic stellate cells in pancreatic cancer metastasis. Am J Pathol. 2010; 177:2585-2596. [PubMed: 20934972]

[26]. Kindler HL, Niedzwiecki D, Hollis D, Sutherland S, Schrag D, Hurwitz H, et al. Gemcitabine plus bevacizumab compared with gemcitabine plus placebo in patients with advanced pancreatic cancer: phase III trial of the Cancer and Leukemia Group B (CALGB 80303). J Clin Oncol. 2010; 28:3617-3622. [PubMed: 20606091] 
[27]. Kindler HL, Wroblewski K, Wallace JA, Hall MJ, Locker G, Nattam S, et al. Gemcitabine plus sorafenib in patients with advanced pancreatic cancer: a phase II trial of the University of Chicago Phase II Consortium. Invest New Drugs. 2012; 30:382-386. [PubMed: 20803052]

[28]. Friess H, Langrehr JM, Oettle H, Raedle J, Niedergethmann M, Dittrich C, et al. A randomized multi-center phase II trial of the angiogenesis inhibitor Cilengitide (EMD 121974) and gemcitabine compared with gemcitabine alone in advanced unresectable pancreatic cancer. BMC Cancer. 2006; 6:285. [PubMed: 17156477]

[29]. Hingorani SR, Petricoin EF, Maitra A, Rajapakse V, King C, Jacobetz MA, et al. Preinvasive and invasive ductal pancreatic cancer and its early detection in the mouse. Cancer Cell. 2003; 4:437450. [PubMed: 14706336]

[30]. Aguirre AJ, Bardeesy N, Sinha M, Lopez L, Tuveson DA, Horner J, et al. Activated Kras and Ink4a/Arf deficiency cooperate to produce metastatic pancreatic ductal adenocarcinoma. Genes Dev. 2003; 17:3112-3126. [PubMed: 14681207]

[31]. Bardeesy N, Cheng KH, Berger JH, Chu GC, Pahler J, Olson P, Hezel AF, Horner J, Lauwers GY, Hanahan D, DePinho RA. Smad4 is dispensable for normal pancreas development yet critical in progression and tumor biology of pancreas cancer. Genes Dev. 2006; 20:3130-3146. [PubMed: 17114584]

[32]. Hingorani SR, Wang L, Multani AS, Combs C, Deramaudt TB, Hruban RH, et al. Trp53R172H and KrasG12D cooperate to promote chromosomal instability and widely metastatic pancreatic ductal adenocarcinoma in mice. Cancer Cell. 2005; 7:469-483. [PubMed: 15894267]

[33]. Provenzano, Paolo P.; Cuevas, C.; Chang, Amy E.; Goel, Vikas K.; Von Hoff, Daniel D.; Hingorani, Sunil R. Enzymatic Targeting of the Stroma Ablates Physical Barriers to Treatment of Pancreatic Ductal Adenocarcinoma. Cancer cell. 2012; 21:418-429. [PubMed: 22439937]

[34]. Whipple CA, Young AL, Korc M. A Kras(G12D)-driven genetic mouse model of pancreatic cancer requires glypican-1 for efficient proliferation and angiogenesis. Oncogene. 2012; 31:2535-2544. [PubMed: 21996748]

[35]. DiMagno EP, Buxton JL, Regan PT, Hattery RR, Wilson DA, Suarez JR, Green PS. Ultrasonic endoscope. Lancet. 1980; 1:629-631. [PubMed: 6102631]

[36]. Haycox A, Lombard M, Neoptolemos J, Walley T. Review article: current practice and future perspectives in detection and diagnosis of pancreatic cancer. Aliment Pharmacol Ther. 1998; 12:937-948. [PubMed: 9798798]

[37]. Saftoiu A, Vilmann P. Role of endoscopic ultrasound in the diagnosis and staging of pancreatic cancer. J Clin Ultrasound. 2009; 37:1-17. [PubMed: 18932265]

[38]. Othman MO, Wallace MB. The role of endoscopic ultrasonography in the diagnosis and management of pancreatic cancer. Gastroenterol Clin North Am. 2012; 41:179-188. [PubMed: 22341257]

[39]. Minniti S, Bruno C, Biasiutti C, Tonel D, Falzone A, Falconi M, Procacci C. Sonography versus helical CT in identification and staging of pancreatic ductal adenocarcinoma. J Clin Ultrasound. 2003; 31:175-182. [PubMed: 12692824]

[40]. Hunt GC, Faigel DO. Assessment of EUS for diagnosing, staging, and determining resectability of pancreatic cancer: a review. Gastrointest Endosc. 2002; 55:232-237. [PubMed: 11818928]

[41]. Borbath I, Van Beers BE, Lonneux M, Schoonbroodt D, Geubel A, Gigot JF, Deprez PH. Preoperative assessment of pancreatic tumors using magnetic resonance imaging, endoscopic ultrasonography, positron emission tomography and laparoscopy. Pancreatology. 2005; 5:553561. [PubMed: 16113592]

[42]. Helmstaedter L, Riemann JF. Pancreatic cancer--EUS and early diagnosis. Langenbecks Arch Surg. 2008; 393:923-927. [PubMed: 18247044]

[43]. Harewood GC, Wiersema MJ. Endosonography-guided fine needle aspiration biopsy in the evaluation of pancreatic masses. Am J Gastroenterol. 2002; 97:1386-1391. [PubMed: 12094855]

[44]. Shah JN, Ahmad NA, Beilstein MC, Ginsberg GG, Kochman ML. Clinical impact of endoscopic ultrasonography on the management of malignancies. Clin Gastroenterol Hepatol. 2004; 2:10691073. [PubMed: 15625651]

[45]. Olson P, Chu GC, Perry SR, Nolan-Stevaux O, Hanahan D. Imaging guided trials of the angiogenesis inhibitor sunitinib in mouse models predict efficacy in pancreatic neuroendocrine 
but not ductal carcinoma. Proceedings of the National Academy of Sciences. 2011; 108:E1275E1284.

[46]. Michl P, Pauls S, Gress TM. Evidence-based diagnosis and staging of pancreatic cancer. Best Pract Res Clin Gastroenterol. 2006; 20:227-251. [PubMed: 16549326]

[47]. Nichols MT, Russ PD, Chen YK. Pancreatic imaging: current and emerging technologies. Pancreas. 2006; 33:211-220. [PubMed: 17003640]

[48]. Ellsmere J, Mortele K, Sahani D, Maher M, Cantisani V, Wells W, et al. Does multidetector-row CT eliminate the role of diagnostic laparoscopy in assessing the resectability of pancreatic head adenocarcinoma? Surg Endosc. 2005; 19:369-373. [PubMed: 15624058]

[49]. Muller MF, Meyenberger C, Bertschinger P, Schaer R, Marincek B. Pancreatic tumors: evaluation with endoscopic US, CT, and MR imaging. Radiology. 1994; 190:745-751. [PubMed: 8115622]

[50]. Klau M, Siller W, Pahn G, Fritz F, Kieser M, Werner J, et al. Dual-energy perfusion-CT of pancreatic adenocarcinoma. Eur J Radiol. 2013; 82:208-214. [PubMed: 23062281]

[51]. D’Onofrio M, Galloti A, Mantovani W, Crosara S, Manfrin E, Falconi M, et al. Perfusion CT can predict tumoral grading of pancreatic adenocarcinoma. Eur J Radiol. 2013; 82:227-233. [PubMed: 23127804]

[52]. Gambhir SS, Czernin J, Schwimmer J, Silverman DH, Coleman RE, Phelps ME. A tabulated summary of the FDG PET literature. J Nucl Med. 2001; 42:1S-93S. [PubMed: 11483694]

[53]. Keogan MT, Tyler D, Clark L, Branch MS, McDermott VG, DeLong DM, Coleman RE. Diagnosis of pancreatic carcinoma: role of FDG PET. AJR Am J Roentgenol. 1998; 171:15651570. [PubMed: 9843289]

[54]. Yokoyama Y, Nagino M, Hiromatsu T, Yuasa N, Oda K, Arai T, et al. Intense PET signal in the degenerative necrosis superimposed on chronic pancreatitis. Pancreas. 2005; 31:192-194. [PubMed: 16025008]

[55]. Fendrich V, Schneider R, Maitra A, Jacobsen ID, Opfermann T, Bartsch DK. Detection of precursor lesions of pancreatic adenocarcinoma in PET-CT in a genetically engineered mouse model of pancreatic cancer. Neoplasia. 2011; 13:180-186. [PubMed: 21403843]

[56]. Serrano OK, Chaudhry MA, Leach SD. The role of PET scanning in pancreatic cancer. Adv Surg. 2010; 44:313-325. [PubMed: 20919529]

[57]. Diederichs CG, Staib L, Vogel J, Glasbrenner B, Glatting G, Brambs HJ, et al. Values and limitations of 18F-fluorodeoxyglucose-positron-emission tomography with preoperative evaluation of patients with pancreatic masses. Pancreas. 2000; 20:109-116. [PubMed: 10707924]

[58]. Vachiranubhap B, Kim YH, Balci NC, Semelka RC. Magnetic resonance imaging of adenocarcinoma of the pancreas. Top Magn Reson Imaging. 2009; 20:3-9. [PubMed: 19687720]

[59]. Kamisawa T, Takuma K, Anjiki H, Egawa N, Hata T, Kurata M, et al. Differentiation of autoimmune pancreatitis from pancreatic cancer by diffusion-weighted MRI. Am J Gastroenterol. 2010; 105:1870-1875. [PubMed: 20216538]

[60]. Weissleder R, Mahmood U. Molecular imaging. Radiology. 2001; 219:316-333. [PubMed: 11323453]

[61]. Weissleder R, Pittet MJ. Imaging in the era of molecular oncology. Nature. 2008; 452:580-589. [PubMed: 18385732]

[62]. Bouvet M, Wang J, Nardin SR, Nassirpour R, Yang M, Baranov E, Jiang P, Moossa AR, Hoffman RM. Real-Time Optical Imaging of Primary Tumor Growth and Multiple Metastatic Events in a Pancreatic Cancer Orthotopic Model. Cancer research. 2002; 62:1534-1540. [PubMed: 11888932]

[63]. Kuchimaru T, Kadonosono T, Tanaka S, Ushiki T, Hiraoka M, Kizaka-Kondoh S. In vivo imaging of HIF-active tumors by an oxygen-dependent degradation protein probe with an interchangeable labeling system. PLoS One. 2010; 5:e15736. [PubMed: 21203417]

[64]. Eser S, Messer M, Eser P, von Werder A, Seidler B, Bajbouj M, et al. In vivo diagnosis of murine pancreatic intraepithelial neoplasia and early-stage pancreatic cancer by molecular imaging. Proc Natl Acad Sci U S A. 2011; 108:9945-9950. [PubMed: 21628592] 

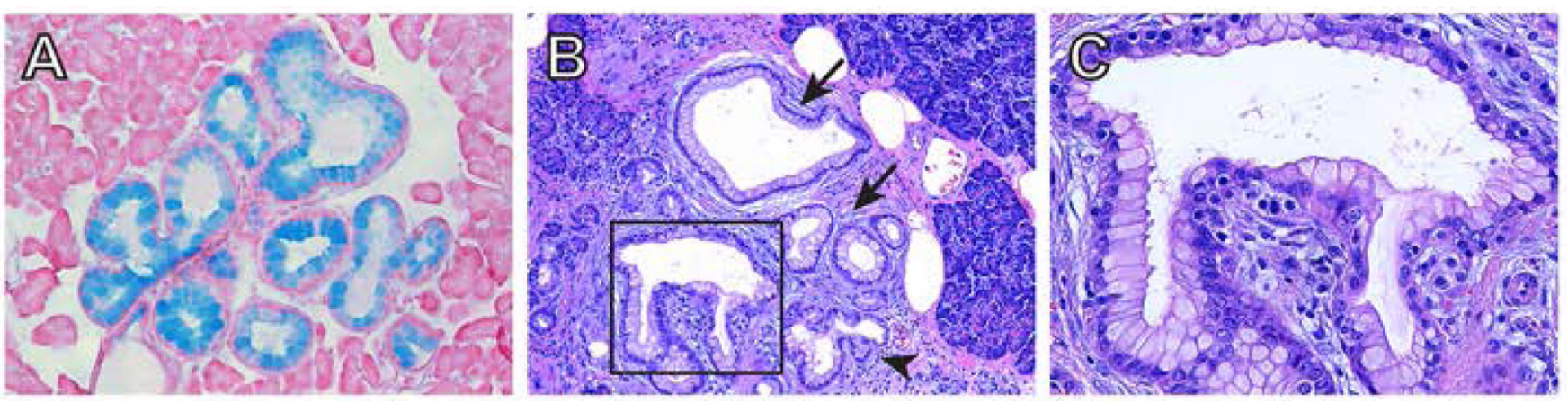

Fig.1.

PanIN Lesions. (A) PanIN-1 lesions accumulate mucins, evidenced by Alcian blue staining. (B) H\&E stain of human pancreas showing PanIN-1A (arrows) and PanIN-1B (arrowhead) lesions adjacent to a PanIN-2 (boxed). (C) High magnification image of the PanIN-2 lesion in (B) showing luminal budding, and nuclear elongation and loss of polarity. 


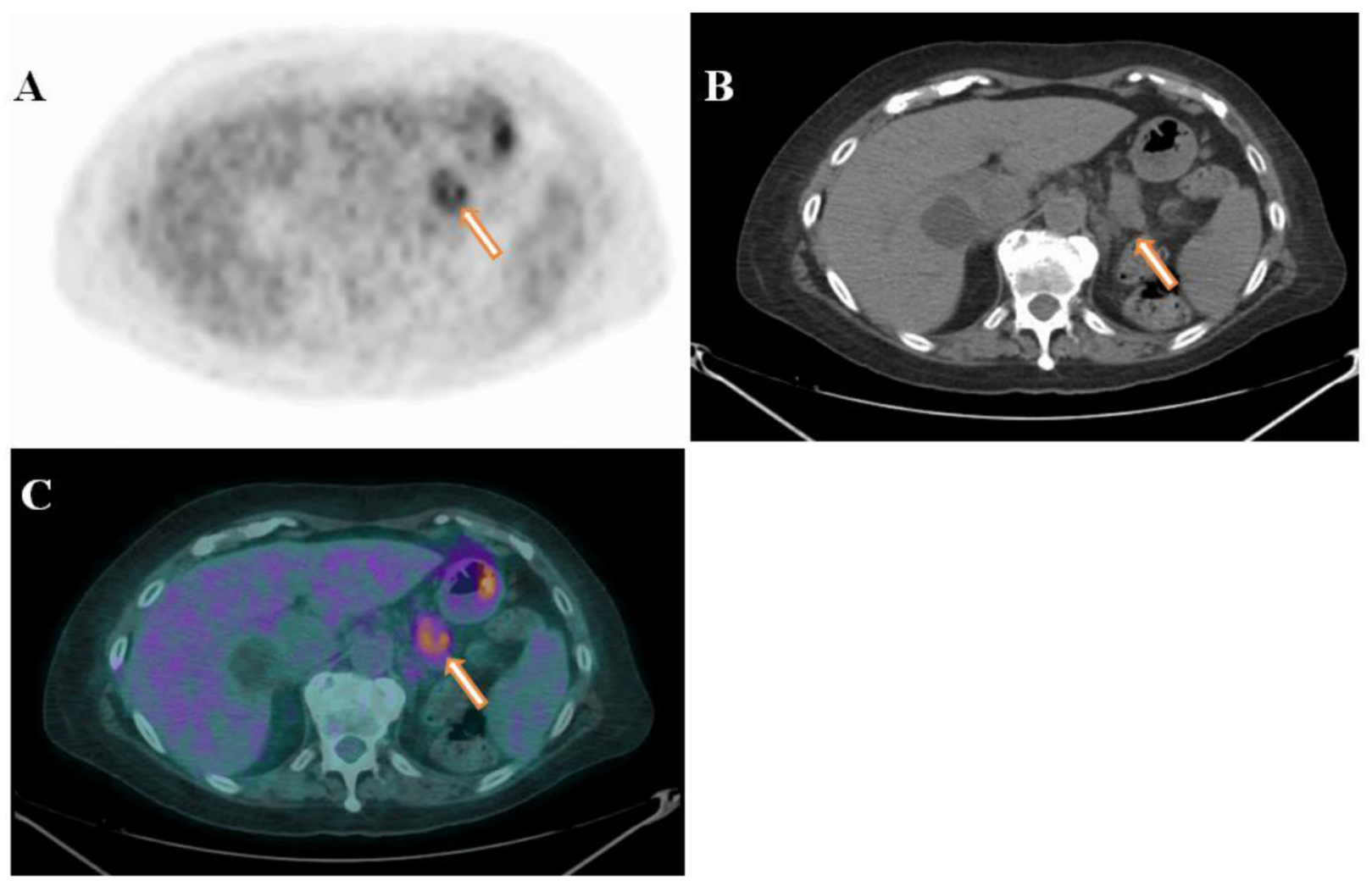

Fig.2.

Axial PET (A), CT (B), and fused PET/CT (C) images of a 78 year-old female with a hypermetabolic mass (SUVmax 3.9) in the tail of the pancreas. The image is compatible with primary PDAC (arrows). Figure courtesy of Dr. Gregory Ravizzini from MD Anderson Cancer Center. 


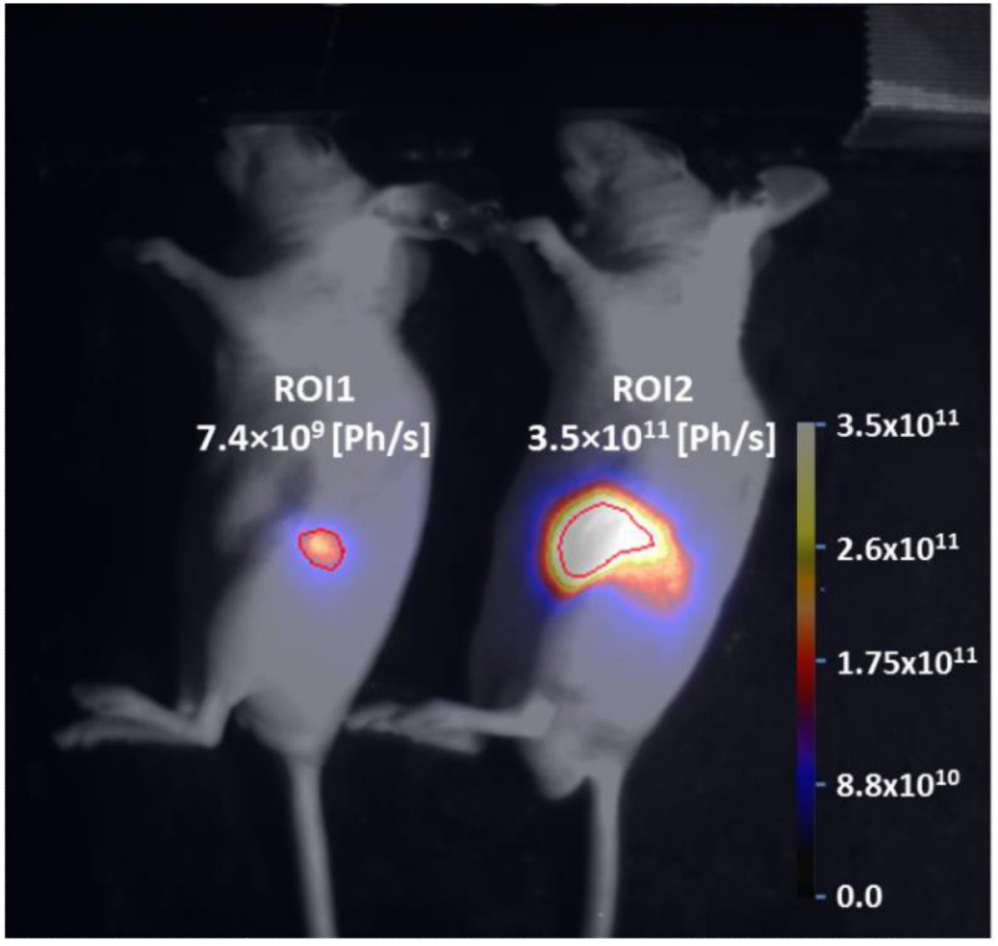

Fig.3.

BLI of pancreatic T3M4-Luc tumors in a mouse orthotopic model. Mice were injected with $1.5 \times 10^{5} \mathrm{~T} 3 \mathrm{M} 4-\mathrm{Luc}$ cells and imaged 21 days later. Regions of interest (ROI) show integrated light signal in both tumors scaled to the maximum value and measured in photons/second $(\mathrm{Ph} / \mathrm{s})$. 
Table 1

A summary of the most commonly used imaging modalities.

\begin{tabular}{|c|c|c|c|c|c|}
\hline Imaging Modality & Resolution & $\begin{array}{c}\text { Sensitivity } \\
\text { [Moles/l] }\end{array}$ & Imaging Agents & Cost & $\begin{array}{c}\text { Clinical/ } \\
\text { Animal Use }\end{array}$ \\
\hline PET & $\begin{array}{c}1-2 \mathrm{~mm} \\
\text { (microPET); } \\
4-5 \mathrm{~mm}(\mathrm{cllinical} \\
\text { PET }\end{array}$ & $\begin{array}{c}\sim 10^{-} 11-10^{-} \\
12\end{array}$ & $\begin{array}{c}\text { FDG, FLT, choline, } \\
\text { acetate, } \\
\text { nitroimidazole, } \\
\text { antibody and } \\
\text { antibody fragments }\end{array}$ & High & Yes/Yes \\
\hline CT & $15-200 \mathrm{~m}$ & $\sim 10^{-4}$ & $\begin{array}{c}\text { Iodine } \\
\text { Barium }\end{array}$ & High & $\begin{array}{c}\text { Yes/bone and } \\
\text { lung imaging }\end{array}$ \\
\hline MRI & $10-100 \mathrm{~m}$ & $\sim 10^{-3}-10^{-5}$ & $\begin{array}{c}\text { Gadolinium }(+3) \text {, iron } \\
\text { oxide particles, } \\
\text { manganese oxide }\end{array}$ & High & Yes/Yes \\
\hline Ultrasound & $>40 \mathrm{~m}$ & $\begin{array}{c}\text { a single } \\
\text { bubble }\end{array}$ & $\begin{array}{c}\text { Contrast microbubles } \\
\text { Low }\end{array}$ & Yes/Yes \\
\hline $\begin{array}{c}\text { Bioluminescence } \\
\text { Fluorescence }\end{array}$ & $2-3 \mathrm{~mm}$ & $\sim 10^{-15}$ & $\begin{array}{c}\text { Fluorescent } \\
\text { molecules and dyes, } \\
\text { targeted } \\
\text { nanoparticles }\end{array}$ & Low & No/Yes \\
\hline
\end{tabular}

